\title{
Detección de fluorescencia en sistemas modelo conteniendo aldehidos: relación con la alteración de pescado
}

\author{
Por Santiago P. Aubourg \\ Instituto de Investigaciones Marinas (CSIC). C/. Eduardo Cabello, 6. 36208 - VIGO. \\ e-mail saubourg@iim.csic.es
}

\section{RESUMEN}

Detección de fluorescencia en sistemas modelo conteniendo aldehidos: relación con la alteración de pescado.

En estudios previos se llevó a cabo la detección de compuestos de interacción a través de sus propiedades fluorescentes a distintos máximos de excitación/emisión durante el procesamiento de pescado, observándose un desplazamiento de la fluorescencia a longitudes de onda mayores como resultado del incremento de la alteración. En el presente trabajo se chequearon distintos tipos de aldehidos al objeto de comparar su capacidad para formar compuestos fluorescentes de distintas longitudes de onda en relación con el procesamiento de pescado. Dichos aldehidos (formaldehido, FA; acetaldehido, AcH; propanal, Pr; hexanal, Hex; 2-hexenal, $\mathrm{Hx}$; benzaldehido, $\mathrm{BzH}$ ) se hicieron reaccionar con músculo de sardina (Sardina pilchardus) desmenuzada y con n-propilamina a $30^{\circ} \mathrm{C}$ en oscuridad durante 25 días.

Con el aumento del tiempo de reacción, todos los aldehidos estudiados mostraron capacidad para producir un desplazamiento a mayores longitudes de onda de la fluorescencia detectada. Sin embargo se observaron distintas aptitudes, de acuerdo con la estructura y la reactividad de cada aldehido. El desplazamiento fue especialmente pronunciado en los casos de $\mathrm{Hx}$ y $\mathrm{AcH}$, mientras que fue bastante pequeño con FA y BzH. En una situación intermedia se situaron $\mathrm{Pr}$ y Hex, en los que al igual que durante la alteración de pescado, se observó un incremento en la relación entre las fluorescencias detectadas a $393 / 463 \mathrm{~nm}$ y $327 / 415 \mathrm{~nm}$ al avanzar el tiempo de reacción.

PALABRAS-CLAVE: Aldehido - Amina - Fluorescencia Interacción - Oxidación lipídica.

\section{SUMMARY}

Fluorescence detection in aldehyde containing model systems: Relationship with fish deterioration.

In previous studies interaction compounds formation was accomplished by fluorescence detection at different excitation/ emission wavelength maxima during fish processing. Afluorescence shift towards higher wavelength maxima was then obtained as a result of damage increase. In the present work, different aldehydes were tested in order to compare their ability in producing fluorescent compounds with similar properties to the ones obtained during fish processing. Aldehydes (formaldehyde, FA; acetaldehyde, $\mathrm{AcH}$; propanal, Pr; hexanal, Hex; 2-hexenal $\mathrm{Hx}$; benzaldehyde, $\mathrm{BzH}$ ) were reacted in model systems with sardine (Sardina pilchardus) muscle and with n-propylamine at $30^{\circ} \mathrm{C}$ in dark during 25 days.

A fluorescence shift was detected for all the aldehydes investigated as long as the reaction time increased. Great differences were observed between aldehydes, according to their chemical structure and reactivity. A sharp shift was detected for $\mathrm{Hx}$ and $\mathrm{AcH}$, while little changes were observed in the cases of $\mathrm{FA}$ and $\mathrm{BzH}$. As in the case of fish processing, $\mathrm{Pr}$ and Hex showed an increasing ratio between fluorescences detected at $393 / 463 \mathrm{~nm}$ and $327 / 415 \mathrm{~nm}$ as long as the reaction time increased.

KEY-WORDS: Aldehyde - Amine - Fluorescence - Interaction - Lipid oxidation.

\section{INTRODUCCIÓN}

El procesamiento de los alimentos puede llevar a la formación de una amplia gama de productos de oxidación lipídica (Hsieh y Kinsella, 1989, Flick et al., 1992). Los aldehidos representan la fracción más importante de los productos de oxidación secundaria, por lo que se han realizado numerosos esfuerzos para su determinación (Tappel, 1980, Esterbauer, 1982, Chiba et al., 1989). Debido a su gran reactividad, los aldehidos tienen facilidad para interaccionar con los constituyentes nucleófilos del tipo amino (proteínas, péptidos, aminoácidos libres, fosfolípidos aminados) presentes en los alimentos produciendo pardeamiento (Pokorny, 1981, Zamora e Hidalgo, 1992), cambios de aroma (Pearson et al., 1977, Yasuhara y Shibamoto, 1995) y pérdidas de nutrientes esenciales (Nielsen et al., 1985, Hidalgo et al., 1992) durante el procesamiento y el almacenamiento.

El método más empleado para la detección de estos compuestos de interacción ha sido el que se basa en sus propiedades fluorescentes. Durante muchos años los estudios de fluorescencia han sido llevados a cabo a un solo máximo de excitación/ emisión (Leake y Karel, 1985, Smith et al., 1990, Lubis y Buckle, 1990), llegándose a considerar esta medición como una herramienta complementaria de otras medidas más desarrolladas a la hora de determinar el grado de alteración de los alimentos (Melton, 1983, Kim y Labella, 1987).

En investigaciones previas realizadas en nuestro equipo se han estudiado las alteraciones de pescado durante el procesamiento a través de distintos valores de fluorescencia de los compuestos de interacción formados (Aubourg et al., 1992a, 1992b, 
1995). En la medida que cada vez se iban estudiando muestras de pescado más alteradas, el máximo de fluorescencia se iba desplazando a longitudes de onda mayores. La determinación de este desplazamiento de la fluorescencia como la relación entre dos de estos máximos de fluorescencia (393/463 nm y $327 / 415$ $\mathrm{nm}$ ) se correlacionó satisfactoriamente con el tiempo de almacenamiento y otros índices de calidad (Aubourg et al., 1996, 1997).

En el presente trabajo se chequearon distintos tipos de aldehidos, al objeto de estudiar su capacidad para formar compuestos de interacción con las mismas propiedades fluorescentes (mismos máximos de excitación/emisión) que las detectadas durante la alteración de pescado. Los ensayos se realizaron en sistemas modelo a $30^{\circ} \mathrm{C}$, empleando $n$-propilamina y músculo de sardina como fuentes de compuestos nucleófilos. A partir de los resultados se discuten factores como la longitud de la cadena y grado de insaturación del aldehido empleado, así como el tiempo de reacción.

\section{PARTE EXPERIMENTAL}

En el desarrollo del presente trabajo se emplearon reactivos de grado analítico (E. Merck, Darmstadt, R. F. Alemana).

\subsection{Sistemas modelo aldehido-músculo de pescado}

Tres docenas de sardina fresca (Sardina pilchardus) se dividieron en tres lotes, al objeto de estudiarlos separadamente. Dentro de cada lote, el músculo blanco de sardina se separó y desmenuzó. Asimismo, se prepararon disoluciones 0 '5 $\mathrm{M}$ de etanol acuoso al $86 \%$ de cada uno de los siguientes aldehidos: formaldehido (FA), acetaldehido $(\mathrm{AcH})$, propanal (Pr), hexanal (Hex), 2-hexenal $(\mathrm{Hx})$ y benzaldehido $(\mathrm{BzH})$.

Se mezclaron porciones de sardina desmenuzada $(4 \mathrm{~g})$ con $10 \mathrm{ml}$ de disolución de cada uno de los aldehidos. Las mezclas de reacción se mantuvieron a $30^{\circ} \mathrm{C}$ en oscuridad en tubos cerrados y a tiempos definidos (1, 7 y 25 días) se sometieron al análisis por fluorescencia. Paralelamente se analizaron blancos consistentes en cada uno de los reactivos de partida (músculo de sardina y aldehidos) que fueron sometidos a las mismas condiciones de reacción.

\subsection{Sistemas modelo aldehido-propilamina}

Se preparó una disolución 0'1 M de n-propilamina (Pam) y disoluciones 0'5 M de cada uno de los alde- hidos (FA, AcH, $\mathrm{Pr}, \mathrm{Hex}, \mathrm{Hx}$ y BzH) en etanol acuoso al $86 \%$.

Diez $\mathrm{ml}$ de disolución de cada aldehido se mezclaron con $10 \mathrm{ml}$ de disolución de propilamina y con $10 \mathrm{ml}$ de etanol acuoso al $86 \%$. Las mezclas se incubaron a $30^{\circ} \mathrm{C}$ en oscuridad en tubos cerrados y a tiempos definidos ( 1,7 y 25 días) se sometieron al análisis por fluorescencia. Las mezclas de reacción se llevaron a cabo por triplicado a lo largo de todo el experimento. Asimismo se analizaron blancos consistentes en los reactivos de partida (Pam y aldehidos) que fueron sometidos a las mismas condiciones de reacción.

\subsection{Análisis de fluorescencia}

Las mezclas de reacción conteniendo músculo de pescado fueron centrifugadas $(3000 \mathrm{~g}$ durante 10 minutos) y filtradas. La parte líquida resultante se expuso a la luz UV ( $350 \mathrm{~nm}$ ) durante 30 s con el fin de destruir cualquier resto de retinol presente en el extracto, y posteriormente fue analizada por fluorescencia. En el caso de los sistemas modelo conteniendo propilamina, las mezclas de reacción se analizaron directamente.

Los espectros de excitación y emisión de las mezclas de reacción se estudiaron en un espectrómetro de fluorescencia Perkin-Elmer LS 3B. Las medidas se realizaron en los siguientes máximos de excitación/ emisión: $327 / 415$ nm, 393/463 nm, 479/516 nm y $528 / 556 \mathrm{~nm}$.

Como estándar de fluorescencia se empleó una disolución de sulfato de quinina $(1 \mu \mathrm{g} / \mathrm{ml}$ en sulfúrico 0 '05 $M)$. Los valores de fluorescencia se calcularon como la relación: $F / F_{s t}$, donde $F$ es la fluorescencia del medio de reacción medida a cada máximo de longitud de onda, y $F_{\mathrm{st}}$ es la fluorescencia del estándar en el correspondiente máximo. El valor $\delta \mathrm{F}$ se calculó como la relación entre los valores de fluorescencia a $393 / 463 \mathrm{~nm}$ y $327 / 415 \mathrm{~nm}$. Las medidas de fluorescencia se realizaron a temperatura ambiente $\left(16-18^{\circ} \mathrm{C}\right)$ con cubetas de cuarzo de $10 \mathrm{~mm}$ de paso de luz.

\subsection{Análisis estadístico}

Los datos correspondientes a los valores de fluorescencia y de $\delta \mathrm{F}$ se sometieron al análisis de varianza de una vía (Statsoft, 1994). La significación se declaró a $\mathrm{p}<0$ 0'05.

\section{RESULTADOS}

Los medios de reacción correspondientes a blancos de aldehidos no experimentaron cambios signifi- 
cativos a lo largo del experimento en ninguno de los máximos de fluorescencia considerados. Por tanto, la evaluación de resultados se centra en las mezclas de reacción donde los aldehidos se encuentran en presencia de sustancias amínicas (propilamina o incluidas en el músculo de sardina).

\subsection{Sistemas modelo aldehido-músculo de Pescado (Tabla I)}

En el caso del blanco de músculo de sardina se observa un aumento progresivo en los tres máximos a lo largo del tiempo, como resultado de la presencia tanto de productos de oxidación lipídica como de sustancias nucleófilas. El mismo resultado se obtiene para el FA, con la diferencia que el incremento fue mucho mayor en los dos máximos de fluorescencia mayores $(393 / 463 \mathrm{~nm}$ y $479 / 516 \mathrm{~nm})$.

Tabla I

Valores de fluorescencia

$(327 / 415 \mathrm{~nm}, 393 / 463 \mathrm{~nm} \text { y } 479 / 516 \mathrm{~nm})^{*}$ en los sistemas modelo aldehido - músculo de sardina **

\begin{tabular}{|c|c|c|c|c|}
\hline $\begin{array}{l}\text { Mezcla de } \\
\text { Reacción }\end{array}$ & $\begin{array}{c}\text { Tiempo de } \\
\text { Reacción (dias) }\end{array}$ & $327 / 415 \mathrm{~nm}$ & $393 / 463 \mathrm{~nm}$ & $479 / 516 \mathrm{~nm}$ \\
\hline MS & 0 & $0,38 \mathrm{a}$ & $0,39 \mathrm{a}$ & $4,94 \mathrm{a}$ \\
\hline MS & 1 & 0 '67 b & $0,35 \mathrm{a}$ & $7 ' 26 a$ \\
\hline MS & 7 & $0,89 \mathrm{c}$ & $0^{\prime} 57 \mathrm{~b}$ & $5,26 a$ \\
\hline MS & 25 & $1 ' 77 d$ & $2 ' 14 \mathrm{c}$ & $10^{\prime} 87 \mathrm{~b}$ \\
\hline $\mathrm{FA}$ & 0 & $0,10 \mathrm{a}$ & $0,12 \mathrm{a}$ & $0^{\prime} 35 \mathrm{a}$ \\
\hline$M S+F A$ & 1 & $0^{\prime} 25 \mathrm{~b}$ & $0,37 a$ & $5^{\prime} 15 b$ \\
\hline $\mathrm{MS}+\mathrm{FA}$ & 7 & $0^{\prime} 44 \mathrm{c}$ & $1^{\prime} 53 \mathrm{~b}$ & $7 ' 15 \mathrm{c}$ \\
\hline$M S+F A$ & 25 & $0,64 \mathrm{~d}$ & $435 c$ & $13^{\prime} 41 \mathrm{~d}$ \\
\hline $\mathrm{AcH}$ & 0 & $0^{\prime} 10 \mathrm{~b}$ & $0,12 \mathrm{a}$ & $0^{\prime} 33 \mathrm{a}$ \\
\hline $\mathrm{MS}+\mathrm{AcH}$ & 1 & $0,23 \mathrm{c}$ & 1 '05 b & $700 \mathrm{~b}$ \\
\hline $\mathrm{MS}+\mathrm{AcH}$ & 7 & $0,02 \mathrm{a}$ & $5,30 \mathrm{c}$ & $28 ' 93 \mathrm{c}$ \\
\hline $\mathrm{MS}+\mathrm{AcH}$ & 25 & 0'01 a & $4^{\prime} 86 \mathrm{c}$ & $36 ' 83 d$ \\
\hline $\mathrm{Pr}$ & 0 & $0,10 a$ & $0,12 \mathrm{a}$ & $0^{\prime} 34 \mathrm{a}$ \\
\hline$M S+P r$ & 1 & $0^{\prime} 20 \mathrm{~b}$ & $0^{\prime} 78 \mathrm{~b}$ & $5^{\prime} 59 \mathrm{~b}$ \\
\hline$M S+P r$ & 7 & 0'14 a & $1 ' 47 c$ & $7 ' 55 c$ \\
\hline$M S+P r$ & 25 & $0^{\prime} 15 \mathrm{a}$ & $3744 d$ & $9^{\prime} 24 \mathrm{~d}$ \\
\hline Hex & 0 & $0^{\prime} 10 a$ & $0^{\prime} 15 a$ & $0^{\prime} 33 \mathrm{a}$ \\
\hline $\mathrm{MS}+\mathrm{Hex}$ & 1 & $0^{\prime} 28 \mathrm{c}$ & $0^{\prime} 72 \mathrm{~b}$ & $5 ' 80 \mathrm{~b}$ \\
\hline MS + Hex & 7 & $0^{\prime} 22 \mathrm{~b}$ & $1 ' 61 \mathrm{c}$ & $7 ' 13 b$ \\
\hline MS + Hex & 25 & $0^{\prime} 26 \mathrm{c}$ & $3^{\prime} 66 \mathrm{~d}$ & $11^{\prime} 48 \mathrm{c}$ \\
\hline $\mathrm{Hx}$ & 0 & $\mathrm{Nd}^{* * *}$ & $0^{\prime} 16 \mathrm{a}$ & $0^{\prime} 39 \mathrm{a}$ \\
\hline $\mathrm{MS}+\mathrm{Hx}$ & 1 & $\mathrm{Nd}$ & $0^{\prime} 83 \mathrm{~b}$ & $37^{\prime} 57 \mathrm{~b}$ \\
\hline $\mathrm{MS}+\mathrm{Hx}$ & 7 & $\mathrm{Nd}$ & $1 / 45 d$ & $85^{\prime} 86 \mathrm{c}$ \\
\hline $\mathrm{MS}+\mathrm{Hx}$ & 25 & $\mathrm{Nd}$ & $107 \mathrm{c}$ & $136^{\prime} 08 d$ \\
\hline $\mathrm{BzH}$ & 0 & $\mathrm{Nd}$ & $0,01 \mathrm{a}$ & $0^{\prime} 11 \mathrm{a}$ \\
\hline $\mathrm{MS}+\mathrm{BzH}$ & 1 & $\mathrm{Nd}$ & $0^{\prime} 29 \mathrm{~b}$ & $6^{\prime} 09 \mathrm{~b}$ \\
\hline $\mathrm{MS}+\mathrm{BzH}$ & 7 & $\mathrm{Nd}$ & $0^{\prime} 42 \mathrm{~b}$ & $5^{\prime} 98 \mathrm{~b}$ \\
\hline $\mathrm{MS}+\mathrm{BzH}$ & 25 & Nd & $1 ' 13 \mathrm{c}$ & $9^{\prime} 19 \mathrm{c}$ \\
\hline
\end{tabular}

El AcH muestra un comportamiento muy distinto en los tres máximos de fluorescencia. Después del primer día de reacción se observa un gran descenso de la fluorescencia a $327 / 415 \mathrm{~nm}$. El máximo $393 / 463 \mathrm{~nm}$ refleja un incremento de la fluorescencia detectada hasta el séptimo día, a partir del cual se mantiene en un valor constante hasta el final del experimento. Finalmente, 479/516 nm muestra un incremento constante con el tiempo.

Los aldehidos $\operatorname{Pr}$ y Hex reflejan una respuesta muy similar entre sí. A $327 / 415 \mathrm{~nm}$ hay un comportamiento irregular con el tiempo de reacción, aunque no se producen grandes variaciones. Sin embargo en las otras dos determinaciones se obtiene un incremento constante en la medida que aumenta el tiempo de reacción. En el caso del BzH también se observa un incremento de fluorescencia en los dos máximos mayores, mientras que no se detecta fluorescencia a $327 / 415 \mathrm{~nm}$.

En lo que respecta al $\mathrm{Hx}$ tampoco se detecta fluorescencia a $327 / 415 \mathrm{~nm}$. A $393 / 463 \mathrm{~nm}$ se observa un incremento hasta el séptimo día de reacción, que es seguido por un descenso al final del experimento. Sin embargo a $479 / 516 \mathrm{~nm}$ se produce un incremento a lo largo de toda la experiencia.

\subsection{Sistemas modelo aldehido-propilamina (Tabla II)}

Tanto el blanco de Pam como el sistema modelo con FA produjeron pocos cambios en la fluorescencia desarrollada. El incremento más significativo es el de la medida a 479/516 nm para ambos tipos de sistema modelo.

Una respuesta muy distinta es la proporcionada por el $\mathrm{AcH}$ y el $\mathrm{Hx}$. Ambos aldehidos experimentan un incremento de fluorescencia en los tres máximos en las primeras fases de la interacción, que es seguido por un descenso en la medida que se continúa el curso de la reacción. La desaparición de compuestos fluorescentes es especialmente grande a $327 / 415 \mathrm{~nm}$ y $393 / 463 \mathrm{~nm}$, llegándose a valores no detectables.

Los aldehidos $\operatorname{Pr}$ y Hex mostraron unos resultados muy similares entre sí. A $327 / 415 \mathrm{~nm}$ se observa un incremento en las primeras fases de la interacción seguido de un descenso posterior, a medida que avanza el tiempo de reacción. Sin embargo, los otros dos máximos experimentan un incremento a lo largo de toda la reacción. Un resultado relativamente similar es el observado para el caso del $\mathrm{BzH}$, aunque los valores son claramente inferiores a los de los otros dos aldehidos. 
Tabla II

Valores de fluorescencia $(327 / 415 \mathrm{~nm}, 393 / 463 \mathrm{~nm} \text { y } 479 / 516 \mathrm{~nm})^{*}$ en los sistemas modelo aldehido - propilamina **

\begin{tabular}{|c|c|c|c|c|}
\hline $\begin{array}{c}\text { Mezcla de } \\
\text { Reacción }\end{array}$ & $\begin{array}{c}\text { Tiempo de } \\
\text { Reacción (dias) }\end{array}$ & $327 / 415 \mathrm{~nm}$ & $393 / 463 \mathrm{~nm}$ & $479 / 516 \mathrm{~nm}$ \\
\hline Pam & 0 & 0,09 a & $0,19 \mathrm{c}$ & $0,34 a b$ \\
\hline Pam & 1 & $0^{\prime} 09 \mathrm{a}$ & $0^{\prime} 14 \mathrm{ab}$ & $0^{\prime} 40 \mathrm{~b}$ \\
\hline Pam & 7 & $0,09 \mathrm{a}$ & $0^{\prime} 14 \mathrm{a}$ & $0,32 a$ \\
\hline Pam & 25 & $0,11 \mathrm{a}$ & $0^{\prime} 18 \mathrm{bc}$ & $0^{\prime} 50 \mathrm{c}$ \\
\hline FA & 0 & $0^{\prime} 10 \mathrm{a}$ & $0^{\prime} 12 \mathrm{a}$ & $0,36 \mathrm{a}$ \\
\hline $\mathrm{FA}+\mathrm{Pam}$ & 1 & $0,17 \mathrm{c}$ & $0,14 \mathrm{~b}$ & $0^{\prime} 37 \mathrm{a}$ \\
\hline $\mathrm{FA}+\mathrm{Pam}$ & 7 & $0^{\prime} 14 \mathrm{~b}$ & $0^{\prime} 15 \mathrm{~b}$ & $0,38 \mathrm{a}$ \\
\hline$F A+P a m$ & 25 & o'14 b & $0^{\prime} 14 \mathrm{~b}$ & $0^{\prime} 48 \mathrm{~b}$ \\
\hline $\mathrm{AcH}$ & 0 & $0^{\prime} 10 \mathrm{a}$ & $0^{\prime} 12 \mathrm{a}$ & $0,33 \mathrm{a}$ \\
\hline $\mathrm{AcH}+\mathrm{Pam}$ & 1 & $0^{\prime} 16 \mathrm{~b}$ & $66^{\prime} 50 \mathrm{c}$ & $59^{\prime} 79 \mathrm{~b}$ \\
\hline $\mathrm{AcH}+\mathrm{Pam}$ & 7 & $\mathrm{Nd}^{* * *}$ & 1'53 b & $174^{\prime} 38 \mathrm{c}$ \\
\hline $\mathrm{AcH}+\mathrm{Pam}$ & 25 & $\mathrm{Nd}$ & Nd & $54^{\prime} 37 \mathrm{~b}$ \\
\hline $\mathrm{Pr}$ & 0 & $0^{\prime} 10 \mathrm{a}$ & $0^{\prime} 12 \mathrm{a}$ & $0^{\prime} 34 \mathrm{a}$ \\
\hline $\mathrm{Pr}+\mathrm{Pam}$ & 1 & $0^{\prime} 65 \mathrm{~b}$ & $5^{\prime} 39 \mathrm{~b}$ & $0^{\prime} 46 \mathrm{a}$ \\
\hline $\mathrm{Pr}+\mathrm{Pam}$ & 7 & $0^{\prime} 96 \mathrm{c}$ & $31^{\prime} 81 \mathrm{c}$ & $279 \mathrm{~b}$ \\
\hline $\mathrm{Pr}+\mathrm{Pam}$ & 25 & $0^{\prime} 63 \mathrm{~b}$ & $47^{\prime} 22 \mathrm{~d}$ & $8^{\prime} 91 \mathrm{c}$ \\
\hline Hex & 0 & $0^{\prime} 10 \mathrm{a}$ & $0^{\prime} 16 \mathrm{a}$ & $0^{\prime} 33 \mathrm{a}$ \\
\hline Hex + Pam & 1 & $0^{\prime} 49 \mathrm{~b}$ & 0'99 b & $0^{\prime} 49 \mathrm{~b}$ \\
\hline $\mathrm{Hex}+\mathrm{Pam}$ & 7 & $0^{\prime} 93 \mathrm{~d}$ & $14^{\prime} 80 \mathrm{c}$ & $1 ' 11 \mathrm{c}$ \\
\hline Hex + Pam & 25 & $0776 \mathrm{c}$ & $37^{\prime} 81 \mathrm{~d}$ & $3^{\prime} 84 \mathrm{~d}$ \\
\hline $\mathrm{Hx}$ & 0 & $0^{\prime} 02 \mathrm{a}$ & $0^{\prime} 16 \mathrm{a}$ & $0,39 a$ \\
\hline $\mathrm{Hx}+\mathrm{Pam}$ & 1 & $0^{\prime} 03 \mathrm{~b}$ & $2^{\prime} 42 \mathrm{c}$ & $220^{\prime} 10 \mathrm{~d}$ \\
\hline $\mathrm{Hx}+\mathrm{Pam}$ & 7 & $\mathrm{Nd}$ & $0^{\prime} 37 \mathrm{~b}$ & $125^{\prime} 02 \mathrm{c}$ \\
\hline $\mathrm{Hx}+\mathrm{Pam}$ & 25 & $\mathrm{Nd}$ & $\mathrm{Nd}$ & $35^{\prime} 73 \mathrm{~b}$ \\
\hline $\mathrm{BzH}$ & 0 & $\mathrm{Nd}$ & 0'01 a & $0^{\prime} 03 \mathrm{a}$ \\
\hline $\mathrm{BzH}+\mathrm{Pam}$ & 1 & $\mathrm{Nd}$ & $0^{\prime} 12 \mathrm{~b}$ & $0^{\prime} 02 \mathrm{~b}$ \\
\hline $\mathrm{BzH}+\mathrm{Pam}$ & 7 & Nd & $0^{\prime} 30 \mathrm{c}$ & $0^{\prime} 32 \mathrm{a}$ \\
\hline $\mathrm{BzH}+\mathrm{Pam}$ & 25 & $\mathrm{Nd}$ & $0^{\prime} 63 d$ & $0^{\prime} 47 \mathrm{c}$ \\
\hline
\end{tabular}

* Valores medios de tres determinaciones independientes. En cada columna y para cada reactivo (aldehidos y propilamina), los valores seguidos de letras distintas son sig-

nificativamente distintos ( $\mathrm{p}<0.05)$. panal), Hex (hexanal) $\mathrm{Hx}$ (2-hexanal) y BzH (benzaldehido)

$* * *$ Nd: No detectado.

\section{DISCUSIÓN}

El presente estudio proporciona una comparación entre distintos tipos de aldehidos a la hora de producir los compuestos fluorescentes detectados durante el procesamiento de pescado (refrigeración, cocción y esterilización) (Aubourg et al., 1992b, 1995, 1996, 1997). Como comportamiento general de los aldehidos estudiados, se observó un desplazamiento a longitudes de onda mayores de la fluorescencia producida de acuerdo con la formación progresiva de compuestos de adición con pesos moleculares y número de insaturaciones cada vez mayores (Gardner, 1979, Pokorny, 1977; Kikugawa y Beppu, 1987). Acelerados por la temperatura y la reactividad de los aldehidos, los compuestos fluorescentes formados en las primeras fases de la interacción dan lugar a la formación de compuestos fluorescentes con máxi- mos de excitación/emisión de mayores longitudes de onda que sus precursores, obteniéndose de esta manera un desplazamiento de la fluorescencia detectada.

Se ha comprobado que los aldehidos insaturados y portadores de funciones oxigenadas llevan a la formación de un alto contenido en compuestos fluorescentes (Esterbauer et al., 1986, Montfoort et al., 1987). De acuerdo con estos resultados, en la presente experiencia el $\mathrm{Hx}$ ha experimentado el mayor desplazamiento de fluorescencia hacia longitudes de onda mayores, tanto en presencia de músculo de sardina como de Pam. Otro aldehido que ha producido un gran desplazamiento de fluorescencia ha sido el $\mathrm{AcH}$. En este caso la explicación se puede encontrar en su facilidad para la autocondensación y posterior interacción con compuestos nucleófilos (Fujimoto y Kaneda, 1973) llevando al desarrollo de pardeamiento (Suyama y Adachi, 1979) y de fluorescencia (Aubourg et al., 1992a).

Dado el gran desplazamiento de fluorescencia a longitudes de onda mayores obtenido en ambos casos $(\mathrm{Hx}$ y $\mathrm{AcH})$, se consideró oportuno el estudio de un máximo de fluorescencia adicional $(528 / 556 \mathrm{~nm})$, al objeto de seguir la evolución de los compuestos fluorescentes y por tanto el grado de interacción. Los resultados (Tabla III) conciernen a los sistemas modelo de ambos aldehidos reaccionando con músculo de sardina y con propilamina. Se observó un incremento general hasta el día 7 Después no se observaron diferencias significativas, excepto un aumento en el caso de la mezcla $\mathrm{Hx}+\mathrm{MS}$.

En un trabajo anterior (Montfoort et al., 1987) se estudió la capacidad de distintos aldehidos para formar compuestos fluorescentes al interaccionar con liposomas conteniendo fosfatidiletanolamina, única-

Tabla III

Valores de fluorescencia a $528 / 556 \mathrm{~nm}^{*}$ en sistemas modelo conteniendo acetaldehido y 2-hexenal ${ }^{\star *}$

\begin{tabular}{crrr}
\hline $\begin{array}{c}\text { Mezcla de } \\
\text { Reacción }\end{array}$ & \multicolumn{3}{c}{ Tiempo de Reacción (dias) } \\
\hline & \multicolumn{1}{c}{1} & \multicolumn{1}{c}{25} \\
\cline { 2 - 4 } AcH + Pam & $151^{\prime} 25 \mathrm{a}$ & $284^{\prime} 00 \mathrm{~b}$ & $373^{\prime} 75 \mathrm{~b}$ \\
$\mathrm{Hx}+$ Pam & $390^{\prime} 00 \mathrm{a}$ & $1562^{\prime} 30 \mathrm{~b}$ & $1712^{\prime} 50 \mathrm{~b}$ \\
$\mathrm{AcH}+\mathrm{MS}$ & $13^{\prime} 23 \mathrm{a}$ & $80^{\prime} 40 \mathrm{~b}$ & $85^{\prime} 67 \mathrm{~b}$ \\
$\mathrm{Hx}+\mathrm{MS}$ & $113^{\prime} 67 \mathrm{a}$ & $312^{\prime} 73 \mathrm{~b}$ & $481^{\prime} 67 \mathrm{c}$ \\
\hline
\end{tabular}

* Valores medios de tres determinaciones independientes. Los valores en la misma fila seguidos de letras distintas son significativamente distintos $(p<0.05)$. Los valores iniciales para Pam, MSM, AcH y Hx fueron: 2'68, 7'27, 2'54 y 2'82, respectivamente.

** Abreviaturas: De acuerdo con las Tablas Iy II.

mente se detectó la fluorescencia a un máximo y al comparar los valores obtenidos por los monoaldehidos saturados se concluyó que en la medida que aumentaba su longitud de cadena lo hacía la fluorescencia producida. Este efecto fue atribuido al creciente carácter lipofílico de las cadenas aldehídi- 
cas al aumentar su longitud de cadena. Sin embargo, los presentes resultados han proporcionado conclusiones distintas debido a haber estudiado la fluorescencia a distintos máximos de fluorescencia. Así, se observa que el AcH produce un desplazamiento de la fluorescencia bastante mayor que los aldehidos $\mathrm{Pr}$ y Hex.

Los estudios de fluorescencia realizados en pescado procesado demostraron una interesante correlación entre el estado de calidad y el valor de la relación entre las fluorescencias detectadas a $393 / 463 \mathrm{~nm}$ y $327 / 415 \mathrm{~nm}$. El estudio de dicha relación $(\delta \mathrm{F})$ en la presente experiencia demostró que es en los casos de Pr y Hex donde se mantiene un incremento gradual a lo largo de la reacción tanto en los sistemas modelo con sardina como con Pam. En dichos resultados (Tabla IV) pueden comprobarse los valores superiores del $\mathrm{Pr}$ frente al Hex para ambos tipos de sistema modelo, de acuerdo con la mayor reactividad del primero (Allinger et al., 1971). Estos dos aldehidos ( $\mathrm{Pr}$ y Hex) son conocidos en los estudios de oxidación lipídica por formarse a partir de los ácidos poliinsaturados de las series $\omega 3$ y $\omega 6$, respectivamente (Frankel et al., 1989, Frankel y Tappel, 1991). La determinación de Hex ha sido incluso propuesta como medida del estado de calidad en alimentos (Melton, 1983, Jeon y Bassette, 1984). Los resultados del presente trabajo demuestran una interesante relación entre la presencia de ambos aldehidos y el valor $\delta \mathrm{F}$ encontrado durante la alteración de pescado.

Dentro del procesamiento de especies marinas, el FA es conocido por formarse durante la congelación de gádidos, llevando a la formación de enlaces inter e intramoleculares en cadenas proteicas (Castell et

Tabla IV

Valores $\delta F^{*}$ obtenidos por reacción de propanal y hexanal con $n$-propilamina y músculo de sardina **

\begin{tabular}{cccc}
\hline $\begin{array}{c}\text { Mezcla de } \\
\text { Reacción }\end{array}$ & \multicolumn{3}{c}{ Tiempo de Reacción (dias) } \\
\hline & 1 & 7 & 25 \\
\cline { 2 - 4 } $\operatorname{Pr}+$ Pam & $8^{\prime} 34 \mathrm{a}$ & $33^{\prime} 05 \mathrm{~b}$ & $75^{\prime} 13 \mathrm{c}$ \\
$\mathrm{Pr}+\mathrm{MS}$ & $4^{\prime} 03 \mathrm{a}$ & $10^{\prime} 23 \mathrm{~b}$ & $24^{\prime} 93 \mathrm{c}$ \\
$\mathrm{Hex}+\mathrm{Pam}$ & $2^{\prime} 01 \mathrm{a}$ & $15^{\prime} 95 \mathrm{~b}$ & $49^{\prime} 52 \mathrm{c}$ \\
Hex $+\mathrm{MS}$ & $2^{\prime} 61 \mathrm{a}$ & $7^{\prime} 38 \mathrm{~b}$ & $14^{\prime} 17 \mathrm{c}$ \\
\hline
\end{tabular}

* $\delta F$ : Relación de valores de fluorescencia medidos a $393 / 463 \mathrm{~nm}$ y $327 / 415 \mathrm{~nm}$. Valores medios de tres determinaciones independientes. Los valores en la misma fila seguidos de letras distintas son significativamente distintos $(p<0.05)$. Los valores iniciales de $P$ Hex, Pam y MS fueron: 1'27, 1'58, 2'06 y 1'03, respectivamente.

** Abreviaturas: De acuerdo con las Tablas I y II.

al., 1973, Sotelo et al., 1995). A pesar de su alta reactividad, la Tabla II demuestra su baja capacidad para formar compuestos fluorescentes del tipo de los detectados durante la alteración de pescado. Sin embargo, la Tabla I demuestra que en presencia de otros productos de oxidación producidos a partir del músculo de sardina, dichos compuestos fluorescentes pueden formarse.

Por su parte el BzH ha demostrado una capacidad muy baja para formar compuestos fluorescentes en los máximos estudiados tanto en los sistemas con músculo de sardina como con propilamina. La razón puede encontrarse en el hecho de que el $\mathrm{BzH}$ es un aldehido poco reactivo, debido a soportar el carbono carbonílico una carga positiva relativamente baja y tener por tanto rebajada su nucleofilicidad (Allinger et al., 1971).

Los presentes resultados demuestran el interés por la detección de fluorescencia a distintos máximos de longitud de onda como medida de la formación de compuestos de interacción entre productos de oxidación lipídica y compuestos amínicos. Durante el procesamiento de alimentos, esta medida puede ser especialmente útil al tratarse de sustratos con un alto contenido en lípidos insaturados y en compuestos aminados, como es el caso de las especies marinas, conocidas por poseer una gran proporción de ácidos grasos poliinsaturados (Ackman, 1989) y de aminoácidos básicos tales como lisina (Navarro, 1991).

\section{AGRADECIMIENTOS}

A D. Óscar Bellón y D. Marcos Trigo por su eficaz colaboración en el desarrollo del presente trabajo, y a la Xunta de Galicia la ayuda financiera otorgada (Proyecto FAIR (CEE) CT 95-111).

\section{BIBLIOGRAFÍA}

Ackman, R. (1989).-—Fatty acids».-En: Marine biogenic lipids, fats and oils. R. Ackman (editor). CRC Press, Boca Raton, FL. Vol. 1, pp. 103-137.

Allinger, N., Cava, M., De Jongh, D., Johnson, C., Lebel, N. y Stevens, C (1971).-Química Orgánica.-Edit. Reverté, S. A., Barcelona, pp. 661-666.

Aubourg, S. y Medina, I. (1997).— «Quality differences assessment in canned sardine (Sardina pilchardus) by fluorescence detection».-J Agric. Food Chem. 45, 3617-3621.

Aubourg, S., Medina, I. y Pérez-Martín, R. (1995).— «A comparison between conventional and fluorescence detection methods of cooking-induced damage to tuna fish lipids".-Z. Lebensm. Unters. Forsch. 200, 252255.

Aubourg, S. y Pérez-Martín, R. (1996).——Formación de compuestos fluorescentes durante el almacenamiento refrigerado de sardina (Sardina pilchardus): comparación con índices de alteración lipídica».-Grasas y Aceites 47, 307-312.

Aubourg, S., Pérez-Martín, R., Medina I. y Gallardo, J. (1992a). - «Fluorescence formation by interaction of albacore (Thunnus alalunga) muscle with acetaldehyde in 
a model system".-J Agric. Food Chem. 40, 18051808.

Aubourg, S., Pérez-Martín, R., Medina, I. y Gallardo, J. (1992b).- «Fluorescence formation during albacore (Thunnus alalunga) thermal processing".-Z. Lebensm. Unters. Forsch. 195, 332-335.

Castell, C., Smith, B. y Dyer, W. (1973)._- Effects of formaldehyde on salt extractable proteins of gadoid muscle".-J Fish. Res. Board Can. 30, 1205-1213.

Chiba, T., Takazawa, M. y Fujimoto, K. (1989).— «A simple method for estimating carbonyl content in peroxidecontaining oils".-J Amer. Oil Chem. Soc 66, 15881592.

Esterbauer, H. (1982). - «Aldehydic products of lipid peroxidation».En: Free radicals, lipid peroxidation and cancer. D. McBrien y T. Slater (editores). Academic Press, London, pp. 101-128.

Esterbauer, H., Koller, E., Slee, R. y Koster, F. (1986)."Possible involvement of the lipid peroxidation product 4-hydroxynonenal in the formation of fluorescent chromolipids".-Biochem. J 239, 405-409.

Flick, G., Hong, G. y Knobl, G. (1992).—«Lipid oxidation of seafood during storage».-En: Lipid Oxidation in Food. A St Angelo (editor). Amer. Chem. Soc., Washington DC (USA), pp. 103-137.

Frankel, E, Hu, M. y Tappel, A. (1989).—«Rapid headspace gas chromatography of hexanal as a measure of lipid peroxidation in biological samples".-Lipids 24, 976981.

Frankel, E. y Tappel, A. (1991).—- «eadspace gas chromatography of volatile lipid peroxidation products from human red blood cell membranes".-Lipids 26, 479-484.

Fujimoto, K. y Kaneda, T. (1973).— - «tudies on the brown discoloration of fish products. $V$ Reaction mechanism in the early stage».-Bull. Jpn. Soc. Sci. Fish. 39, 185190.

Gardner, H. (1979)._- «Lipid hydroperoxide reactivity with proteins and amino acids: A review».-J. Agric. Food Chem. 27, 220-229.

Hidalgo, F., Zamora, R. y Girón, J. (1992)._«Modificaciones producidas en las proteínas alimentarias por su interacción con lípidos peroxidados. III. Consecuencias nutricionales y toxicológicas".-Grasas y Aceites 43, 97-100.

Hsieh, R. y Kinsella, J. (1989). - "Oxidation of polyunsaturated fatty acids: mechanisms, products, and inhibition with emphasis on fish".-Adv. Food Res. Nutr Res. 33, 233-341.

Jeon, I. y Bassette, R. (1984).- - «Analysis of $n$-pentanal and $n$-hexanal as indices of potato chip shelf-life".- J Food Qual. 7, 97-105.

Kikugawa, K. y Beppu, M. (1987).—«Involvement of lipid oxidation products in the formation of fluorescent and cross-linked proteins".-Chem. Phys. Lipids 44, 277-297.

Kim, R. y Labella, F. (1987)._ «Comparison of analytical methods for monitoring autoxidation profiles of authentic lipids».—J Lipid Res. 28, 1110-1117.
Leake, L. y Karel, M. (1985).— «Nature of fluorescent compounds generated by exposure of protein to oxidizing lipids".-J Food Biochem. 9, 117-136.

Lubis, Z. y Buckle, K. (1990).- «Rancidity and lipid oxidation of dried-salted sardines".- Int. J Food Sci. Technol. 25, 295-303.

Melton, S. (1983).— - Methodology for following lipid oxidation in muscle foods".-Food Technol. 37, 105$111,116$.

Montfoort, A., Bezstarosti, K., Groh, M. y Koster, J. (1987)."The influence of the chain length of aldehydes on the fluorescence of chromolipids".-FEBS Lett 226, 101104.

Navarro, P. (1991)._- «Valor nutritivo del pescado. I Pescado fresco".-Rev. Agroq. Tecnol. Alim. 31, 330-342.

Nielsen, H., Finot, P. y Hurrell, R. (1985). - «Reactions of proteins with oxidizing lipids. 1 Analytical measurements of lipid oxidation and of amino acid losses in a whey protein-methyl linolenate model system".-Brit. J. Nutrit. 53, 7586.

Pearson, A., Love, J. y Shorland F. (1977).- «Warmed-over flavor in meat, poultry and fish".-Adv. Food Res. 23, 2-61.

Pokorny, J. (1977)._- Interaction of oxidized lipids with proteins".-Riv. Ital. Sostanze Grasse 54, 389-393.

Pokorny, J. (1981)._- «rowning from lipid-protein interactions".-Prog. Food Nutr. Sci. 5, 421-428.

Smith, G., Hole, M. y Hanson, S. (1990)._- «Assessment of lipid oxidation in Indonesian salted-dried Marine catfish (Arius thalassinus)».-J. Sci. Food Agric. 51, 193-205.

Sotelo, C., Piñeiro, C. y Pérez-Martín, R. (1995).«Denaturation of fish proteins during frozen storage: role of formaldehyde».-Z Lebensm. Unters. Forsch. 200, 14-23.

Statsoft (1994).- Statistica for Macintosh. Statsoft and its licensors. Tulsa, Oklahoma, USA.

Suyama, K. y Adachi, S. (1979).— - Browning reaction between phospholipids and acetaldehyde».-Agric. Biol. Chem. 43, 1981-1982.

Tappel, A. (1980).— «Measurement of and protection from in vivo lipid peroxidation".-En: Free Radicals in Biology. W Pryor (editor); Academic Press, New York (USA). Vol. 4, pp. 1-47.

Yasuhara, A. y Shibarnoto, T. (1995)._-"Quantitative analysis of volatile aldehydes formed from various kinds of fish flesh during heat treatment».-J. Agric. Food Chem. 43, 94-97.

Zamora, R. e Hidalgo, F. (1992).—-Browning and fluorescence development during microwave irradiation of a lysine/(E)4, 5-epoxy-(E)-2-heptenal model system».-J. Agric. Food Chem. 40, 2269-2273. 